\title{
The RoboNet-II project: Microlensing Anomaly Follow-up with the Las Cumbres Observatory Global Telescope Network (poster)
}

\author{
Yiannis Tsapras* \\ Las Cumbres Observatory \\ E-mail: ytsapraselcogt. net
}

\begin{abstract}
The projected capabilities of LCOGT are driven by the requirements of time-domain astrophysics. In terms of the search for extra-solar planets, LCOGT is active in both transit searches and microlensing event follow-up. The capabilities of the network make it a natural partner for the fully automated approach adopted by the RoboNet-II Consortium. Using the eSTAR intelligent agents[1], we are able to schedule observations dynamically in response to targets selected by the ARTEMiS system [2]
\end{abstract}

The Manchester Microlensing Conference: The 12th International Conference and ANGLES Microlensing Workshop

January 21-25, 2008

Manchester, $U K$

* Speaker. 


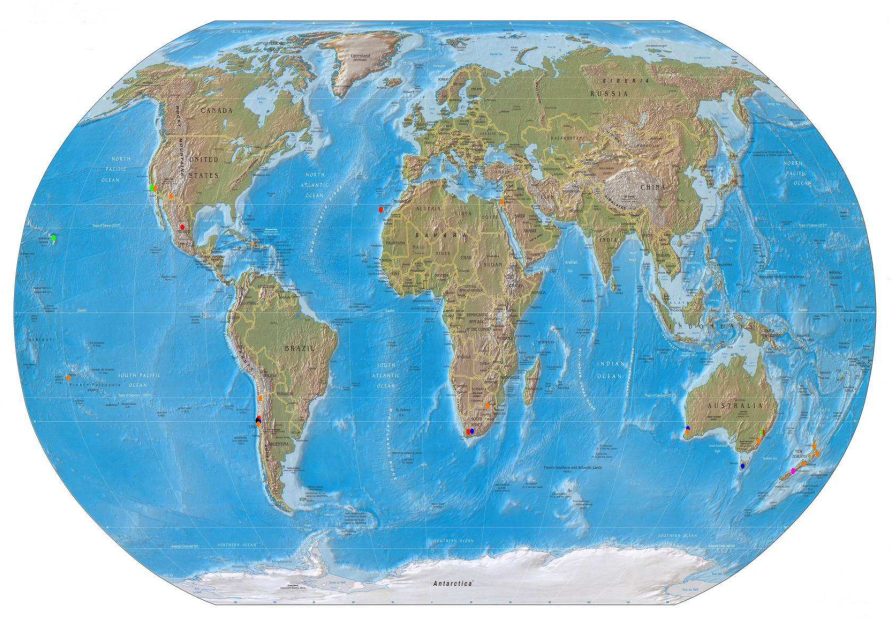

Figure 1: Fig.1 Worldwide Distribution of Observers of Microlensing Events. Red: LCOGT (planned), Green: LCOGT (active), Orange: MicroFUN, Blue: PLANET/RoboNet, Violet: MOA, Black: OGLE

\section{LCOGT deployment Strategy}

LCOGT is building two networks of completely robotic telescopes that will all be linked through the internet. The first network, primarily used for education purposes, will consist of $240.4 \mathrm{~m}$ telescopes.ă The second network, which is intended for science use, will consist of 18 $1.0 \mathrm{~m}$ telescopes which will be deployed in clusters of three telescopes per observing site. There are two major upgrade projects scheduled for the existing $2.0 \mathrm{~m}$ telescopes but there are currently no plans to develop a third one. LCOGT collaborates with other institutions and facilities to best realize it's science and educational goals.

With at total of 44 telescopes planned to come online by 2011, the LCOGT network will be able to provide uninterrupted monitoring of microlensing events.

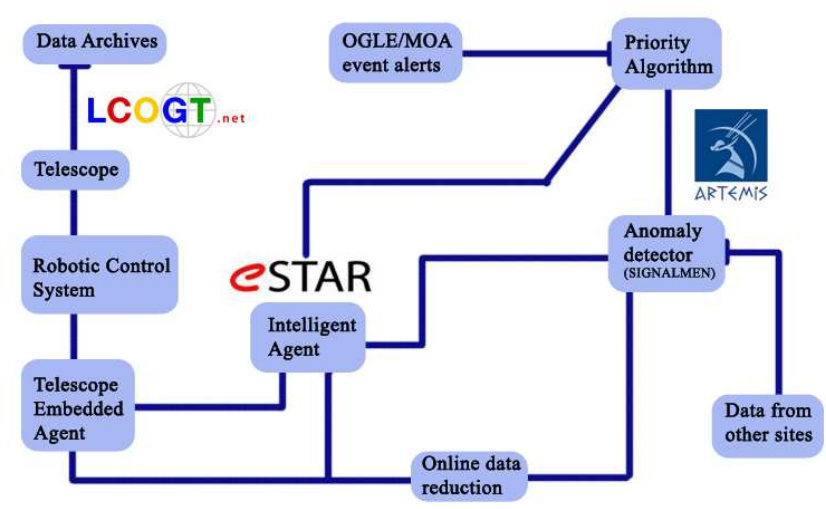

Figure 2: Fig.2 Flowchart of the robotic observing system for the RoboNet-II microlensing campaign. 


\section{Automated Microlensing Observing System}

Microlensing observations with LCOGT telescopes and the Liverpool Telescope are scheduled robotically via eSTAR [1]. Manual overrides are seamlessly integrated to the system. The Incoming images are processed in real time with a difference imaging pipeline, and the data are uploaded to an anomaly detector [2]. Current efforts are directed towards improving the automation and response time of the software chain.

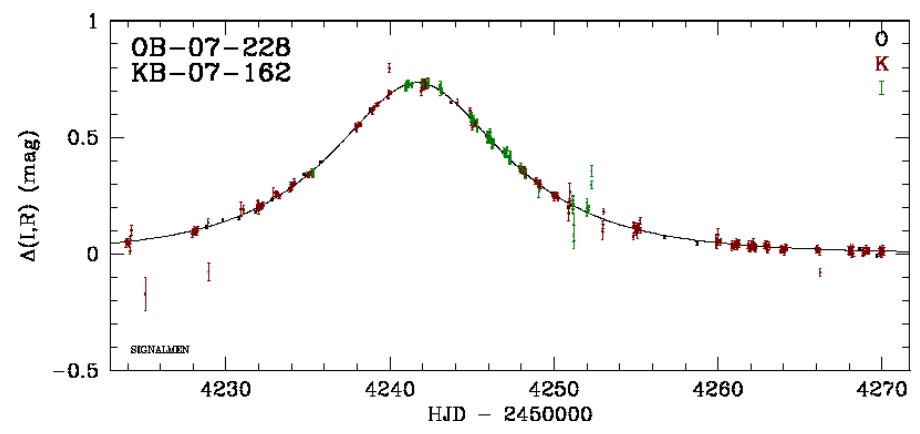

Figure 3: Fig .3 Black $=$ OGLE, Brown $=$ MOA, Green $=$ FTS

A prioritization algorithm is used to scan through the list of all available observable events at any one time and instructs the telescopes on what to follow.

To illustrate the lightcurve coverage, figure 3 shows the sampling obtained for OB07228/KB07162.

Detection maps like the one shown in figure 4 are generated for each event followed and show regions where the data exclude the presence of a planet. In the case that the lightcurve holds evidence of a planetary presence, the exclusion zones (black) would manifest as strongly white detection regions.

\section{Future Plans}

\subsection{8}

- Data reduction \& archiving relocated to LCOGT's base in Santa Barbara.

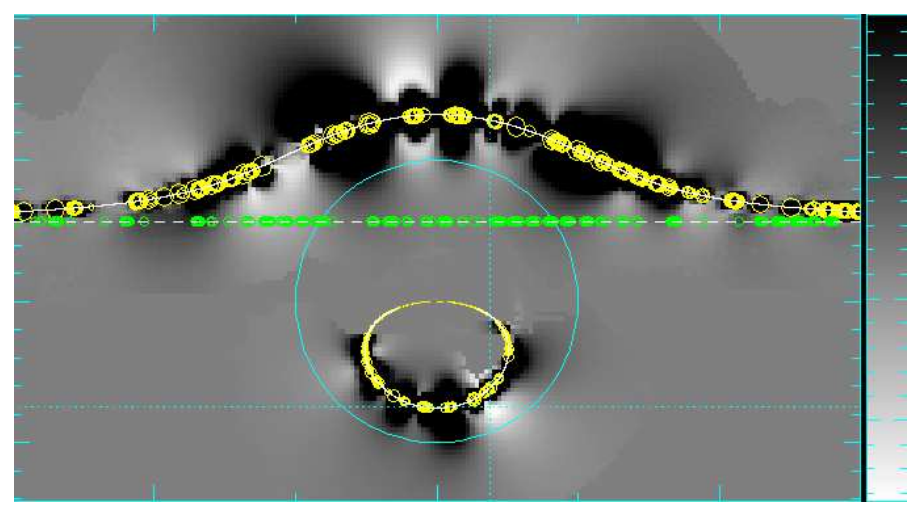

Figure 4: Fig.4 (Non-)Detection $\Delta \chi^{2}$ Map for the event shown in figure 3. 
- Work ongoing to make data flow and response fully automated.

- ARTEMiS system will go online, running the upgraded SIGNALMEN microlensing anomaly detector.

- Restructuring of the priority algorithm.

- Current Hawkcam cameras to be replaced with Spectral Instruments 600 series cameras with Fairchild Imaging ccd486 devices on FTN and FTS, plus extensive upgrades to improve the pointing, tracking and focusing.

- First deployment of $0.4 \mathrm{~m}$ telescopes, development of $1 \mathrm{~m}$ prototype.

\subsection{9}

- Deployment of $0.4 \mathrm{~m}$ and first $1 \mathrm{~m}$ telescopes.

- Development of improved real-time models for microlensing events.

\section{$3.32010-11$}

- Deployment of $1 \mathrm{~m}$ telescopes.

\section{References}

[1] Allan, Naylor, 2006, Proceedings of the HTN Workshop 2005, Steele I., Allan A. (eds), AN, 327, 767. www.estar.org.uk

[2] SIGNALMEN/ARTEMiS: Dominik, et al., 2007, MNRAS, 380, 792. www.artemis-uk.org 